\title{
ABSTRACT
}

Meetings of The Keio Medical Society

\section{Virus and Host Events in Squamous Carcinogenesis}

\author{
Nicholas Coleman \\ Department of Pathology, Cambridge University, Cambridge, United Kingdom
}

Our group investigates early and late stages in the development of squamous cell carcinomas (SCCs), in order to identify new approaches to diagnosis and therapy. The principal themes are:

1. Transcriptional deregulation of high-risk human papillomavirus (HRHPV)

We study mechanisms of HRHPV transcriptional deregulation and competitive cell selection during SCC progression, focussing on the causes and effects of integration of HPV16 into the host genome. We showed that HPV16 integration does not necessarily lead to a competitive growth advantage or increased levels of virus oncogenes. Selection of individual cells is determined through multiple layers of epigenetic regulation of the integrated virus genome. We are currently studying whether the epigenetic landscape of integrated HPV16 reflects that of the host genome at the integration site and/or sites of long-range chromatin interactions of the virus DNA. This work may ultimately identify targets for epigenetic therapies in SCCs containing integrated HRHPV.

2. The oncostatin-M receptor (OSMR) in metastasis

The cell-surface oncostatin-M receptor (OSMR) has emerged as an exciting therapeutic target in SCCs. Both copy number gain and over-expression of OSMR have a strong adverse prognostic effect in SCCs. Carcinoma cells that over-expressed OSMR were more sensitive to the major ligand OSM, which induced multiple promalignant effects, including epithelial-mesenchymal transition, increased metastasis and the ability to induce pro-malignant changes in cells from the tumour microenvironment (TME). We are now studying the effectiveness of OSM:OSMR blockade on the growth and metastasis of SCC cells in vivo, as well as effects on the TME in immunocompetent hosts.

(Presented at the 1923th Meeting, August 10, 2016) 